\title{
О подвижности носителей заряда определенной энергии
}

\author{
(С Ю.М. Белоусов, В.Н. Горелкин, И.В. Черноусов \\ Московский фризико-технический институт (государственный университет), \\ 141701 Долгопрудный, Россия \\ E-mail: ichernousov@inbox.ru
}

(Получена 8 сентября 2016 г. Принята к печати 27 апреля 2017 г.)

Рассматривается функция квазиподвижности носителей заряда с заданной энергией для описания их динамики с помощью кинетического уравнения в важном случае изотропного двухмоментного приближения. В стационарном случае функция квазиподвижности не зависит от функции источника носителей и позволяет вычислить интегральную подвижность. Анализируется связь квазиподвижности с параметрами системы. Доказывается, что в общем случае эта характеристика не описывает вклад в подвижность носителей с определенной энергией. Как известно, в случае почти упругого рассеяния понятие квазиподвижности может иметь красивый физический смысл, однако для случая рассеяния носителей на акустических фононах в твердом теле такая интерпретация квазиподвижности оказывается неверной из-за особенностей интеграла столкновений и вида функции квазиподвижности.

DOI: 10.21883/FTP.2018.01.45314.8404

\section{1. Введение}

В последнее десятилетие происходит бурное развитие электроники на основе алмаза, которое связано с уникальными характеристиками этого широкозонного полупроводникового материала: рекордными значениями твердости, скорости звука, теплопроводности, ширины запрещенной зоны, напряженности пробоя, подвижности носителей, устойчивости к радиационному излучению [1]. Такие характеристики позволяют применять созданные на его основе приборы (как пассивные, типа детекторов частиц, так и активные, типа электронных вентилей) в экстремальных условиях высоких температур, сильного радиационного облучения и большого проходящего тока [2-5]. Это делает алмаз перспективным для использования в атомной промышленности, космических исследованиях, экспериментальной физике плазмы и физике высоких энергий, системах радиолокации и связи.

Технологический прогресс позволяет в настоящее время как синтезировать образцы заданных характеристик, высокого качества и повторяемости [2,4], так и надежно создавать на их основе нужные структуры и комбинировать их с элементами из других материалов, в том числе с электрическими контактами.

Как для конструирования приборов с нужными свойствами, так и для разработки новых устройств с уникальными характеристиками необходимо правильно описывать и рассчитывать кинетику движения носителей заряда в алмазе при различных концентрациях и составе примесей в различных внешних полях и при разных геометриях образца. Уникальные свойства алмаза указывают на то, что в нем могут значительно, по сравнению с обычными полупроводниками, проявляться некоторые необычные кинетические эффекты. Так, при исследовании алмаза мюонным методом [6,7] были получены результаты, которые могли объясняться возникновением у носителей заряда абсолютной отрицательной подвижности (АОП), при которой носители в среднем двигаются против приложенного электрического поля [811]. Предполагалось, что возможная АОП в алмазе связана с сильной неупругостью рассеяния носителей заряда на акустических фононах, вызванной рекордной скоростью звука в алмазе, при температурах порядка 10 К [12]. На первом этапе этих исследований в почти упругом, диффузионно-дрейфовом приближении [12] была, следуя [13], введена характеристика носителей с заданной энергией, которая первоначально считалась подвижностью таких носителей. Эта функция при некоторых энергиях частиц оказывалась отрицательной и приводила к возможности существования интегральной АОП. Последовательный учет неупругости актов взаимодействия носителей с фононами сначала в пространственно однородном случае $[14,15]$, а потом и в пространственно неоднородном случае [16-18] показал, что интегральной АОП добиться не удается. Тем не менее оставался открытым вопрос о смысле функции, характеризующей носители данной энергии и могущей принимать отрицательные значения.

\section{2. Кинетическое уравнение Больцмана в двухмоментном приближении и квазиподвижность}

Запишем уравнение Больцмана для функции распределения носителей заряда $f \equiv f(\mathbf{r}, \mathbf{k}, t)$ в электрическом поле в виде:

$$
\begin{aligned}
\partial f / \partial t+\hbar \mathbf{k} / m \cdot \partial f / \partial \mathbf{r} & +e_{q} \mathbf{E} / \hbar \cdot \partial f / \partial \mathbf{k} \\
& =J_{\text {st }}\{f\}+q(\varepsilon)-v_{\text {cap }}(\varepsilon) f,
\end{aligned}
$$

где $e_{q}-$ заряд носителя, $\mathbf{E}-$ самосогласованное электрического поле, $\mathbf{k}$ - волновой вектор носителей 
заряда, $J_{\mathrm{st}}\{f\}$ - интеграл столкновений, $q(\varepsilon)$ плотность источников носителей заряда с энергией $\varepsilon, v_{\text {сар }}(\varepsilon)-$ частота захвата носителей с энергией $\varepsilon=\hbar^{2} k^{2} / 2 m, m-$ эффективная масса носителя заряда.

В пространственно однородном случае второй член в левой части (1) отсутствует, и уравнение упрощается:

$$
\partial f / \partial t+e_{q} \mathbf{E} / \hbar \cdot \partial f / \partial \mathbf{k}=J_{\text {st }}\{f\}+q-v_{\text {cap }} f .
$$

Будем считать внешнее электрическое поле и, следовательно, самосогласованное поле слабыми, когда можно пренебречь квадратичными по нему слагаемыми. В двухмоментном приближении функция распределения представляется в виде суммы первых двух членов разложения по полиномам Лежандра - изотропной (сферически симметричной) и дипольной части ${ }^{1}$ :

$$
f(\mathbf{k}, t)=f_{0}(\varepsilon, t)+(\mathbf{E k}) \varphi(\varepsilon, t) .
$$

Как обычно, $f_{0}(\varepsilon, t)$ нормируется на плотность носителей, а $\varphi(\varepsilon, t)$ - на плотность тока носителей:

$$
\begin{gathered}
n(t)=\int f(\mathbf{k}, t) d \mathbf{k} /(2 \pi)^{3}=C_{f} \int_{0}^{\infty} \sqrt{\varepsilon} f_{0}(\varepsilon, t) d \varepsilon, \\
\mathbf{j}=\frac{2 e_{q}}{3 \hbar} C_{f} \mathbf{E} \int_{0}^{\infty} \varepsilon^{3 / 2} \varphi(\varepsilon, t) d \varepsilon=e_{q} n \mu \mathbf{E},
\end{gathered}
$$

где $C_{f}=(2 m)^{3 / 2} / 4 \pi^{2} \hbar^{3}$,

$$
\mu=\frac{2}{3 \hbar} \int_{0}^{\infty} \varepsilon^{3 / 2} \varphi(\varepsilon, t) d \varepsilon / \int_{0}^{\infty} \sqrt{\varepsilon} f_{0}(\varepsilon, t) d \varepsilon
$$

- подвижность системы носителей заряда.

Уравнение (1) при этом сводится к системе двух уравнений, которая в стационарном случае имеет вид

$$
\begin{gathered}
0=J_{\mathrm{st}}^{(0)}\left\{f_{0}\right\}+q-v_{\text {cap }} \cdot\left\{f_{0}\right\} \\
e_{q} \hbar / m \cdot \partial f_{0} / \partial \varepsilon=J_{\text {st }}^{(1)}\{\varphi\}-v_{\text {cap }} \cdot \varphi
\end{gathered}
$$

где $J_{\mathrm{st}}^{(0)}\{\ldots\}, J_{\mathrm{st}}^{(1)}\{\ldots\}-$ некоторые функционалы.

Функцию $\varphi$, необходимую для нахождения подвижности, получим из (8) с помощью функции Грина $G\left(\varepsilon, \varepsilon_{0}\right)$ :

$$
\varphi(\varepsilon)=-\int_{0}^{+\infty} \frac{e_{q} \hbar}{m} \frac{\partial f_{0}\left(\varepsilon_{0}\right)}{\partial \varepsilon_{0}} G\left(\varepsilon, \varepsilon_{0}\right) d \varepsilon_{0} .
$$

$G\left(\varepsilon, \varepsilon_{0}\right)$ удовлетворяет уравнению

$$
-\delta\left(\varepsilon-\varepsilon_{0}\right)=-J_{\mathrm{st}}^{(1)}\left\{G\left(\varepsilon, \varepsilon_{0}\right)\right\}+v_{\mathrm{cap}}(\varepsilon) G\left(\varepsilon, \varepsilon_{0}\right) .
$$

\footnotetext{
${ }^{1} \mathrm{~B}$ пространственно неоднородных задачах даже в очень слабых полях для описания функции распределения могут быть существенно необходимы члены разложения высших порядков. Также двухмоментное приближение может не давать количественно правильные результаты при сильной неупругости рассеяния [19].
}

Преобразуем выражение для подвижности (6), поменяв порядок интегрирования и выполнив интегрирование по частям. Для интеграла в числителе имеем:

$$
\begin{aligned}
\int_{0}^{\infty} \varepsilon^{3 / 2} \varphi d \varepsilon & =-\int_{0}^{\infty} \varepsilon^{3 / 2}\left[\int_{0}^{+\infty} \frac{e_{q} \hbar}{m} \frac{\partial f_{0}\left(\varepsilon_{0}\right)}{\partial \varepsilon_{0}} G\left(\varepsilon, \varepsilon_{0}\right) d \varepsilon_{0}\right] d \varepsilon \\
= & -\left.\frac{e_{q} \hbar}{m} f_{0}\left(\varepsilon_{0}\right) \int_{0}^{+\infty} \varepsilon^{3 / 2} G\left(\varepsilon, \varepsilon_{0}\right) d \varepsilon\right|_{0} ^{+\infty} \\
& +\frac{e_{q} \hbar}{m} \int_{0}^{+\infty} f_{0}\left(\varepsilon_{0}\right) \frac{\partial}{\partial \varepsilon_{0}}\left[\int_{0}^{+\infty} \varepsilon^{3 / 2} G\left(\varepsilon, \varepsilon_{0}\right) d \varepsilon\right] d \varepsilon_{0} .
\end{aligned}
$$

В разумных практических случаях первое слагаемое обращается в нуль, поэтому

$$
\begin{aligned}
\mu & =\frac{2}{3 \hbar} \int_{0}^{\infty} \varepsilon^{3 / 2} \varphi d \varepsilon / \int_{0}^{\infty} \sqrt{\varepsilon} f_{0} d \varepsilon \\
& =\int_{0}^{\infty} K\left(\varepsilon_{0}\right) \sqrt{\varepsilon_{0}} f_{0}\left(\varepsilon_{0}\right) d \varepsilon_{0} / \int_{0}^{\infty} \sqrt{\varepsilon} f_{0}(\varepsilon) d \varepsilon,
\end{aligned}
$$

где функцию

$$
K\left(\varepsilon_{0}\right)=\frac{2 e_{q}}{3 m} \frac{1}{\sqrt{\varepsilon_{0}}} \int_{0}^{+\infty} \varepsilon^{3 / 2} G\left(\varepsilon, \varepsilon_{0}\right) d \varepsilon
$$

назовем квазиподвижностью.

\section{3. Квазиподвижность и плотность тока}

Введенная выше функция квазиподвижности имеет смысл, отличный от функции реальной подвижности $K_{\text {real }}(\varepsilon)$, определяемой через плотность тока в соответствии с общепринятой формулой:

$$
\begin{aligned}
K_{\text {real }}(\varepsilon) & =d j / d N \\
& =2 C_{f} / 3 \hbar \cdot \varepsilon^{3 / 2} \varphi(\varepsilon) d \varepsilon /\left(C_{f} \sqrt{\varepsilon} f_{0}(\varepsilon) d \varepsilon\right) \\
& =2 / 3 \hbar \cdot \varepsilon \varphi / f_{0} .
\end{aligned}
$$

Из формул (13) и (14) видно, что функции $K(\varepsilon)$ и $K_{\text {real }}(\varepsilon)$ могут иметь разный вид и смысл. Заметим прежде всего, что, в отличие от $K_{\text {real }}(\varepsilon)$ функция $K(\varepsilon)$ не зависит от функции источника носителей $q(\varepsilon)$ и поэтому более универсальна. Действительно, $K(\varepsilon)$ определяется только интегралом столкновений и стоком носителей и не зависит от $q(\varepsilon)$, в то время как вид $K_{\text {real }}(\varepsilon)$ явно определяется $f_{0}(\varepsilon)$, зависящей от $q(\varepsilon)$. При этом, несмотря на произвол в выборе $q(\varepsilon)$, стационарная $f_{0}(\varepsilon)$ не может получиться произвольной, поскольку она также определяется зависимостью скорости захвата от энергии $v_{\text {сар }}(\varepsilon)$. 
В приближении упругих столкновений функция $K(\varepsilon)$ и ее смысл были впервые подробно изучены Хаксли и Кромптоном (см., например, [13]). В приближении времени релаксации

$$
J_{\mathrm{st}}^{(1)}\{\varphi\}=-v_{\mathrm{tr}} \cdot \varphi,
$$

где $v_{\mathrm{tr}}$ - транспортная частота. Тогда (8) принимает вид

$$
e_{q} \hbar / m \cdot \partial f_{0} / \partial \varepsilon=-v_{\text {tot }} \cdot \varphi
$$

где $v_{\text {tot }}(\varepsilon)=v_{\text {tr }}(\varepsilon)+v_{\text {cap }}(\varepsilon)$ - полная частота.

Из (16) и (6) имеем

$$
\mu=-\frac{2 e_{q}}{3 m} \int_{0}^{\infty} \frac{\varepsilon^{3 / 2}}{v_{\text {tot }}(\varepsilon)} \frac{\partial f_{0}(\varepsilon)}{\partial \varepsilon} d \varepsilon / \int_{0}^{\infty} \sqrt{\varepsilon} f_{0}(\varepsilon) d \varepsilon
$$

Следуя [13], проинтегрируем числитель по частям, тогда при разумных предположениях о поведении $v_{\text {tot }}(\varepsilon)$ в 0 и $\infty$ по энергии получим, что

$K(\varepsilon)=\frac{2 e_{q}}{3 m} \frac{1}{\sqrt{\varepsilon}}\left(\frac{\varepsilon^{3 / 2}}{v_{\text {tot }}(\varepsilon)}\right)^{\prime}=\frac{e_{q}}{m} \cdot \frac{1}{v_{\text {tot }}(\varepsilon)}\left(1-\frac{2}{3} \frac{v_{\text {tot }}^{\prime}(\varepsilon)}{v_{\text {tot }}(\varepsilon)} \varepsilon\right)$.

Здесь и далее для краткости $(\ldots)^{\prime} \equiv \partial(..) / \partial \varepsilon$. Заметим, (18) можно также сразу получить из (13) с помощью функции Грина.

Истинная подвижность частиц с определенной энергией в этом случае равна

$$
K_{\text {real }}(\varepsilon)=d j / d N=-\frac{2 e_{q}}{3 m} \frac{\varepsilon}{v_{\text {tot }}(\varepsilon)} \frac{\partial f_{0} / \partial \varepsilon}{f_{0}} .
$$

Видно, что частицы с энергией, при которой $\partial f_{0}(\varepsilon) / \partial \varepsilon>0$, будут иметь отрицательную подвижность (условие реализуется в заведомо неравновесном случае), что может существенно повлиять на интегральную подвижность.

Одна и та же функция квазиподвижности $K(\varepsilon)$ может определяться разными $v(\varepsilon)$. Действительно, пусть две функции $v(\varepsilon)$ и $\tilde{v}(\varepsilon)$ дают одну и ту же $K(\varepsilon)$ :

$$
K(\varepsilon)=\frac{2 e_{q}}{3 m} \frac{1}{\sqrt{\varepsilon}}\left(\frac{\varepsilon^{3 / 2}}{v(\varepsilon)}\right)^{\prime} \equiv \frac{2 e_{q}}{3 m} \frac{1}{\sqrt{\varepsilon}}\left(\frac{\varepsilon^{3 / 2}}{\tilde{v}(\varepsilon)}\right)^{\prime}
$$

Тогда получаем связь между этими частотами:

$$
\begin{gathered}
\left(\varepsilon^{3 / 2} / v(\varepsilon)\right)^{\prime} \equiv\left(\varepsilon^{3 / 2} / \tilde{v}(\varepsilon)\right)^{\prime}, \\
1 / v(\varepsilon) \equiv 1 / \tilde{v}(\varepsilon)+C / \varepsilon^{3 / 2},
\end{gathered}
$$

где $C$ - некоторая константа.

Из (15) и (18) следует правило сложения квазиподвижностей. Пусть есть $N$ независимых физических механизмов, за счет которых происходит рассеяние носителей, каждый из которых определяет свой интеграл столкновений $J^{(i)}\{\varphi\}$ и свою квазиподвижность $K^{(i)}$, $i=\overline{1, N}$. Пусть $K^{(\mathrm{tot})}-$ квазиподвижность в системе, где имеются все $N$ механизмов рассеяния. Нам интересно, как выражается $K^{(\text {tot })}$ непосредственно через $K^{(i)}, i=\overline{1, N}$. В общем случае найти эту связь затруднительно. Однако в случае, когда элементарные акты почти упругие, работает приближение времени релаксации. Будем считать известными транспортные частоты $v^{(i)}, i=\overline{1, N}$ для всех механизмов рассеяния, при этом частоту захвата включим в одну из транспортных частот. Тогда из (15) имеем:

$$
J^{(i)}\{\varphi\}=-v^{(i)} \cdot \varphi, \quad i=\overline{1, N} ; \quad J^{(\text {tot })}\{\varphi\}=-v^{(\text {tot })} \cdot \varphi,
$$

где

$$
\begin{gathered}
\mathcal{v}^{(\text {tot })}=\sum_{i=1}^{N} v^{(i)} ; \quad K^{(i)}(\varepsilon)=\frac{2 e_{q}}{m} \frac{1}{\sqrt{\varepsilon}}\left(\frac{\varepsilon^{3 / 2}}{v^{(i)}}\right) \\
v^{(i)}(\varepsilon)=2 e_{q} \varepsilon^{3 / 2} /\left(3 m H^{(i)}(\varepsilon)\right) .
\end{gathered}
$$

Здесь $H^{(i)}(\varepsilon)=\int_{d^{(i)}}^{\varepsilon} x^{\frac{1}{2}} K^{(i)}(x) d x+C^{(i)}$, а $d^{(i)}, C^{(i)}$ - некоторые постоянные, обеспечивающие, при известной функции $K^{(i)}(x)$, правильное поведение транспортной частоты. Заметим, что в случае $v^{(i)}(\varepsilon) \propto \varepsilon^{\alpha}, 0<\alpha<3 / 2$, можно положить $d^{(i)}=C^{(i)}=0$.

Тогда

$$
\begin{aligned}
K^{(\mathrm{tot})}(\varepsilon) & =\frac{2 e_{q}}{3 m} \frac{1}{\sqrt{\varepsilon}} \frac{\partial}{\partial \varepsilon} \frac{\varepsilon^{3 / 2}}{v^{(\text {tot })}} \\
& =\frac{2 e_{q}}{3 m} \frac{1}{\sqrt{\varepsilon}} \frac{\partial}{\partial \varepsilon}\left\{\varepsilon^{3 / 2} /\left[\sum_{i=1}^{N} 2 e_{q} \varepsilon^{3 / 2} /\left(3 m H^{(i)}\right)\right]\right\} \\
& =\frac{1}{\sqrt{\varepsilon}} \frac{\partial}{\partial \varepsilon}\left\{1 /\left[\sum_{i=1}^{N} 1 / H^{(i)}\right]\right\}
\end{aligned}
$$

Окончательно получаем общую формулу для сложения квазиподвижностей, обусловленных $N$ независимыми механизмами, в приближении времени релаксации:

$$
\begin{aligned}
K^{(\mathrm{tot})}(\varepsilon) & =\frac{1}{\sqrt{\varepsilon}}\left(1 \sum_{i=1}^{N} 1 / H^{(i)}\right)^{\prime} \\
& =\left(\sum_{i=1}^{N} K^{(i)} /\left(H^{(i)}\right)^{2}\right) /\left(\sum_{i=1}^{N} 1 / H^{(i)}\right)^{2}
\end{aligned}
$$

\section{4. Универсальность функции квазиподвижности при рассеянии на акустических фононах в твердых телах}

Рассмотрим теперь случай неупругого рассеяния носителей на акустических фононах в кристаллах в 

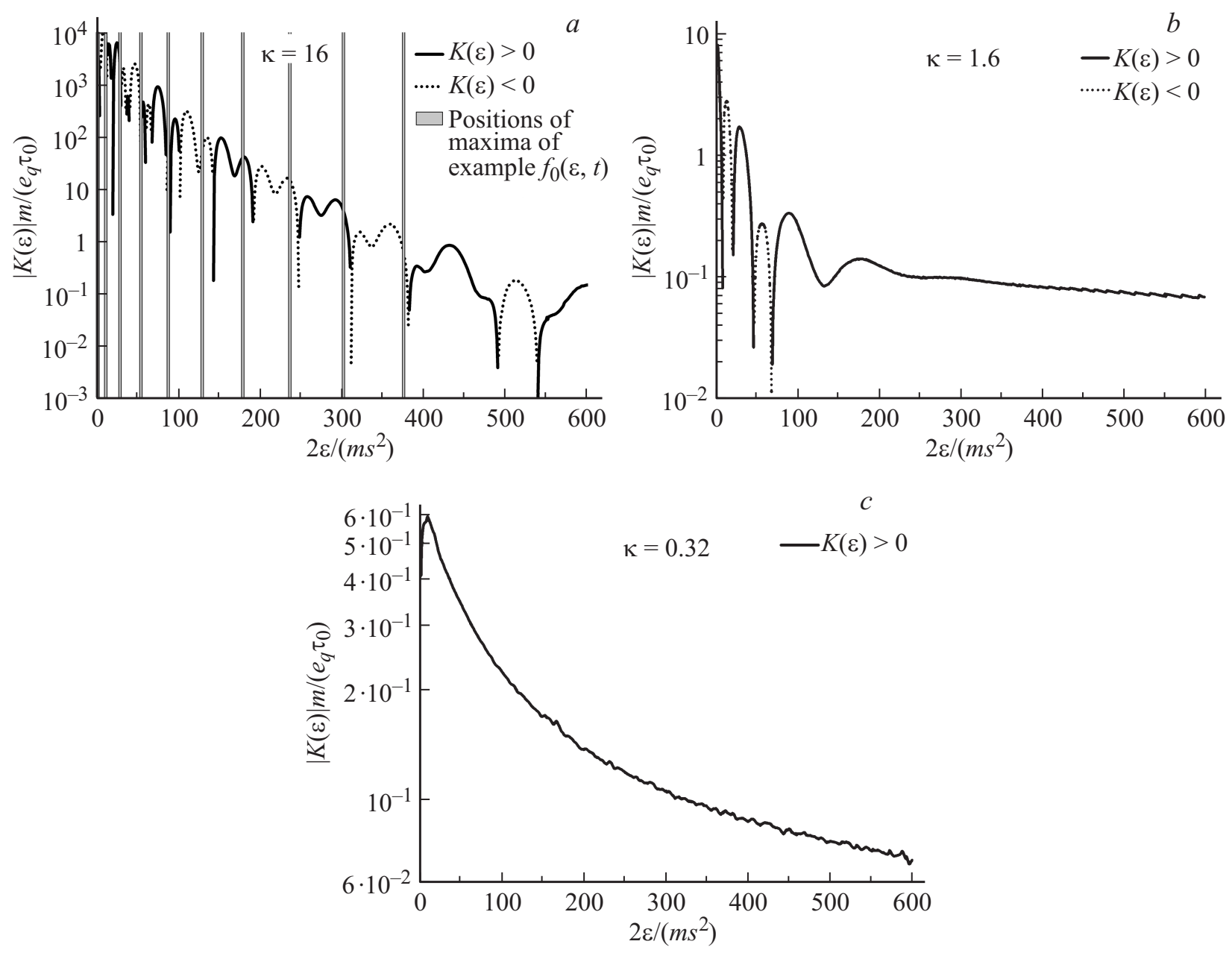

Рис. 1. $a-$ зависимость модуля обезразмеренной квазиподвижности от обезразмеренной энергии при $\kappa=16$. Для иллюстрации „перескоков“ носителей по энергии вертикальными линиями отмечены начальная энергия носителей (крайне правая линия) и энергии, куда в основном переходят носители при испускании фононов; $b$ - зависимость модуля обезразмеренной квазиподвижности от обезразмеренной энергии при $\kappa=1.6 ; c-$ зависимость модуля обезразмеренной квазиподвижности от обезразмеренной энергии при $\kappa=0.32$.

изотропном случае. Выражения для входящих в (7) и (8) интегралов столкновений были получены в [14] в двухмоментном приближении методом потенциала деформации:

$$
\begin{gathered}
J_{\mathrm{st}}^{(0)}\left\{f_{0}\right\}=C(\varepsilon) \int_{q_{0}-q_{\varepsilon}}^{q_{0}+q_{\varepsilon}} \operatorname{sign}(q)\left\{f_{0}(\varepsilon+\hbar s q)\right. \\
\left.\times(\bar{n}(q)+1)-f_{0}(\varepsilon) \bar{n}(q)\right\} q^{2} d q \\
J_{\mathrm{st}}^{(1)}\{\varphi\}=C(\varepsilon) \int_{q_{0}-q_{\varepsilon}}^{q_{0}+q_{\varepsilon}} \operatorname{sign}(q)\{\varphi(\varepsilon+\hbar s q) \\
\left.\times\left[1-\left(\hbar^{2} q^{2} / 2 m-\hbar s q\right) / 2 \varepsilon\right](\bar{n}(q)+1)-\varphi(\varepsilon) \bar{n}(q)\right\} q^{2} d q,
\end{gathered}
$$

где $\quad q_{0}=2 m s / \hbar, \quad q_{\varepsilon}=2 \sqrt{2 m \varepsilon} / \hbar, \quad C(\varepsilon)=\hbar^{2}$ $\times \sqrt{m s^{2} / 2 \varepsilon} /\left(8 \tau_{0} m^{3} s^{3}\right), \quad \tau_{0}=\pi \rho \hbar^{4} / 2 \Xi^{2} m^{3} s$ дает харак- терное время рассеяния носителя на акустическом фононе [14], $\Xi-$ константа деформационного потенциала, $\rho$ - плотность материала, $s-$ скорость звука в кристалле, $\bar{n}(q)=1 /\left(\exp \left(\hbar s q / k_{\mathrm{B}} T\right)-1\right)-$ среднее число фононов с волновым вектором $q$, $T$ - температура кристалла, $k_{\mathrm{B}}-$ постоянная Больцмана, $\hbar s q$ - энергия фонона. Как показано далее, эти формулы справедливы, когда можно пренебречь конечностью температуры Дебая $\Theta_{\mathrm{D}}$.

Интегралы столкновений для $f_{0}$ и $\varphi$ (25), (26) можно переписать в безразмерных переменных, сделав замену: $\varepsilon \rightarrow 2 \varepsilon / m s^{2}, q \rightarrow q / q_{0}, \kappa \rightarrow 2 m s^{2} / k_{\mathrm{B}} T, t \rightarrow t / \tau_{0}$, $\varphi \rightarrow \varphi m^{2} s^{2} /\left(2 e_{q} \hbar \tau_{0}\right)$ :

$$
\begin{aligned}
J_{\mathrm{st}}\left\{f_{0}\right\}= & \frac{1}{\sqrt{\varepsilon}} \int_{1-\sqrt{\varepsilon}}^{1+\sqrt{\varepsilon}} \operatorname{sign}(q)\left\{f_{0}(\varepsilon+4 q, t)(\bar{n}(q)+1)\right. \\
& \left.-f_{0}(\varepsilon, t) \bar{n}(q)\right\} q^{2} d q
\end{aligned}
$$




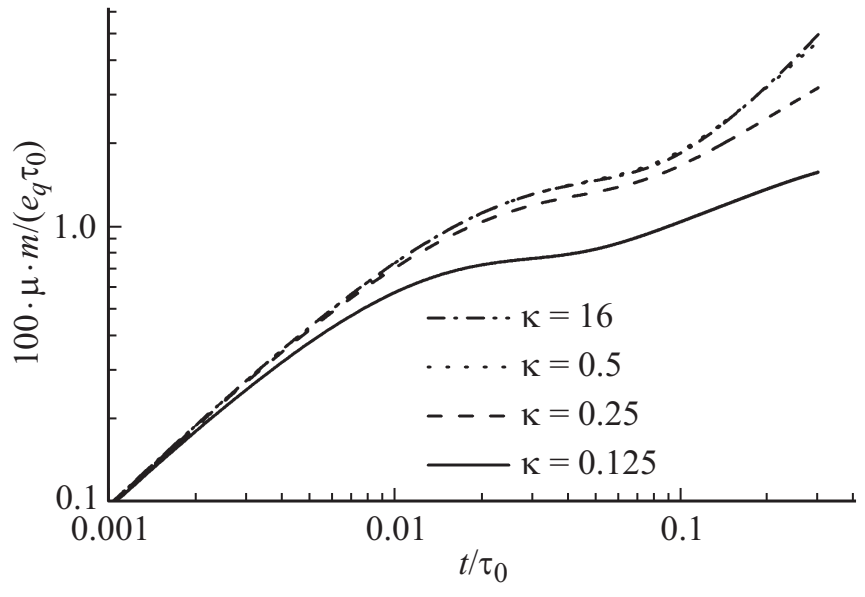

Рис. 2. Зависимость подвижности от времени для носителей с начальной энергией $2 \varepsilon / m s^{2}=125$ при $\kappa=16$ (что соответствует отрицательной квазиподвижности - см. рис. $1, a)$ и при $\kappa \approx 0.5,0.25,0.125$ (для параметров алмаза и носителей, взятых из [15], это соответствует $T=2,64,128$ и $256 \mathrm{~K}$ ).

$$
\begin{aligned}
J_{\text {st }}\{\varphi\}= & \frac{1}{\sqrt{\varepsilon}} \int_{1-\sqrt{\varepsilon}}^{1+\sqrt{\varepsilon}} \operatorname{sign}(q)\left\{\varphi(\varepsilon+4 q, t)\left[1-\frac{2}{\varepsilon}\left(q^{2}-q\right)\right]\right. \\
& \times(\bar{n}(q)+1)-\varphi(\varepsilon, t) \bar{n}(q)\} q^{2} d q
\end{aligned}
$$

В рамках принятых приближений обезразмеривание позволяет получить универсальные для любых материалов зависимости обезразмеренной $K(\varepsilon)$. Опуская громоздкое описание численных расчетов, проведенных при вычислении функции (13), приведем полученные универсальные графики для чистого образца при различных $\kappa$ (рис. 1, $a, b, c)$.

При $\kappa<0.75$ областей отрицательных значений $K(\varepsilon)$ нет (последняя исчезает в районе $2 \varepsilon / m s^{2} \approx 10$ ). При понижении температуры (т.е. при $\kappa \geq 0.75$ ) в области малых энергий начинают возникать осцилляции функции $K(\varepsilon)$, и появляются области отрицательной квазиподвижности. С уменьшением $T$ в районе относительно небольших энергий происходит увеличение абсолютных значений функции $K(\varepsilon)$; ее осцилляции распространяются в область больших энергий. При $\kappa \approx 16$ зоны квазиподвижности определенного знака в области малых энергий начинают разбиваться на отдельные, более узкие зоны.

В работе [13] было показано, что в приближении упругих столкновений квазиподвижность имеет смысл подвижности ансамбля носителей, имеющих фиксированную энергию, вычисленную через среднее смещение в пространстве после большого числа актов рассеяния носителей. При этом считалось, что энергия носителей меняется только за счет работы электрического поля при абсолютно упругом рассеянии.
Возникает вопрос - можно ли в случае рассеяния носителей заряда в твердом теле на акустических фононах приписывать $K(\varepsilon)$ такой физический смысл.

Рассмотрим эволюцию ансамбля носителей, имеющих определенную начальную энергию, с помощью временно́го уравнения Больцмана [15] в чистом образце. График подвижности, полученной в каждый момент времени, показан на рис. 2.

Ни одна из кривых не демонстрирует ни АОП, ни даже дифференциальной отрицательной подвижности, хотя, например, для $\kappa=16$ начальная энергия носителей дает отрицательную квазиподвижность.

\section{5. Обсуждение результатов}

Оказывается, что причиной неприменимости физической интерпретации $K(\varepsilon)$, введенной в [13], в данном случае является существенная неупругость рассеяния на акустических фононах и, самое главное, слишком узкие по энергии зоны квазиподвижности определенного знака.

Действительно, пусть в начальный момент времени все частицы имеют определенную энергию: $f_{0}(\varepsilon)=C \cdot \delta\left(\varepsilon-\varepsilon_{0}\right)$. Для такого распределения выражение (27) принимает вид:

$J_{\text {st }}\left\{f_{0}\right\}=\left\{\begin{array}{l}C \cdot \operatorname{sign}(z)(\bar{n}(z)+1) z^{2} /\left(4 \sqrt{\varepsilon_{0}-4 z}\right), \quad \varepsilon \in I \\ 0, \quad \varepsilon \neq I .\end{array}\right.$

Здесь

$$
I=\left(\left(\sqrt{\varepsilon_{0}}-2\right)^{2} ;\left(\sqrt{\varepsilon_{0}}+2\right)^{2}\right), \quad z=\left(\varepsilon_{0}-\varepsilon\right) / 4,
$$

$\bar{n}(z)=1 /(\exp (\kappa z)-1)$. Таким образом, за один элементарный акт носитель с энергией $\varepsilon_{0}$ может менять ее в пределах „области перескока“ $I$, что следует напрямую из законов сохранения энергии и квазиимпульса (см. рис. 3).

Заметим, что функция $g(z)=\operatorname{sign}(z)(\bar{n}(z)+1) z^{2}$ определяет поведение (29) при всех энергиях, кроме значений, близких к нулю, где поведение интеграла столкновений сильно зависит от множителя $1 / \sqrt{\varepsilon_{0}-4 z} \equiv 1 / \sqrt{\varepsilon}$. Функция $g(z)$ не меняет знак при изменении знака $z$ из-за множителя $\operatorname{sign}(z)$. Асимптотики $g(z)$ :

$$
\begin{array}{ll}
\kappa z \rightarrow 0: & g(z) \rightarrow z \operatorname{sign}(z) / \kappa, \\
\kappa z \rightarrow+\infty: & g(z) \rightarrow z^{2}, \\
\kappa z \rightarrow-\infty: & g(z) \rightarrow z^{2} \exp (-\kappa|z|) .
\end{array}
$$

Левой границе „области перескока“ $\left(\sqrt{\varepsilon_{0}-2}\right)^{2}$ соответствует $z=\sqrt{\varepsilon_{0}}-1$, правой границе „области перескока“ $\left(\sqrt{\varepsilon_{0}+2}\right)^{2}$ соответствует $z=\sqrt{\varepsilon_{0}}+1$, поэтому интеграл столкновений будет стремиться на левой и правой границах „области перескока“ к указанным выше асимптотическим значениям при условиях 


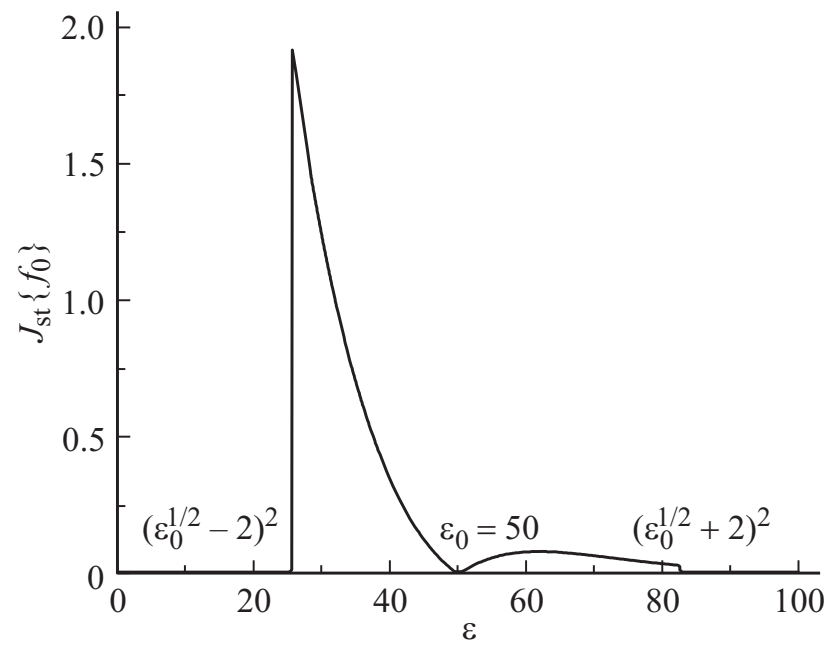

Рис. 3. Интеграл столкновений $J_{\text {st }}\left\{f_{0}\right\}$ для симметричной части функции распределения при $f_{0}(\varepsilon)=C \cdot \delta\left(\varepsilon-\varepsilon_{0}\right), \varepsilon=50$, $\kappa=0.5, C=1$. Видно, что частицы с наибольшей вероятностью переходят на левую границу „области перескока“.

$\kappa\left(\sqrt{\varepsilon_{0}}-1\right)>1, \kappa\left(\sqrt{\varepsilon_{0}}+1\right)>1$ соответственно. Можно показать, что при таких условиях значение $J_{\mathrm{st}}$ на левой границе будет максимальным по сравнению со значениями при любых других энергиях (см. рис. 3).

Таким образом, за один акт частицы из начального положения $\varepsilon_{0}$ переходят в основном в область левой границы $\left(\sqrt{\varepsilon_{0}-2}\right)^{2}$. За следующий акт значительная часть этих частиц перейдет в область $\left(\sqrt{\left(\sqrt{\varepsilon_{0}}-2\right)^{2}}-2\right)^{2}$ и т. п., за $n$ актов заметное число частиц прорелаксирует до энергии $\left(\sqrt{\varepsilon_{0}}-2 n\right)^{2}$. Таким образом, эти частицы уменьшат свою энергию относительно исходной $\varepsilon_{0}$ на

$$
\begin{aligned}
\Delta(n) & =\varepsilon_{0}-\left(\sqrt{\varepsilon_{0}}-2 n\right)^{2}=4 n\left(\sqrt{\varepsilon_{0}}-n\right) \\
& =-4\left(n-\sqrt{\varepsilon_{0}} / 2\right)^{2}+\varepsilon_{0},
\end{aligned}
$$

значит, примерно за $\left[\sqrt{\varepsilon_{0}} / 2\right]$ актов они полностью потеряют энергию. Например, тяжелым дыркам в кремнии $\left(m s^{2} / k_{\mathrm{B}} \approx 3 \mathrm{~K}\right)$ с начальной энергией 0.5 эВ может понадобиться всего примерно 20 актов, чтобы достичь нулевой энергии.

Описанная особенность динамики частиц в энергетическом пространстве проясняет проблему с физической интерпретацией $K(\varepsilon)$, предложенной в [13]. Действительно, пусть $\kappa=16$, и все частицы имеют начальную энергию, равную $375 \mathrm{~ms}^{2} / 2$. На рис. 1 , а вертикальными линиями отмечена энергия исходного ансамбля носителей (крайне правая линия) и энергии, в которые преимущественно будут переходить носители при испускании фононов. Эти энергии определяются полученной выше формулой $\left(\sqrt{\varepsilon_{0}}-2 n\right)^{2}$. Легко видеть, что размер отдельного перескока по энергии $\Delta(1)=4\left(\sqrt{\varepsilon_{0}}-1\right)$ соизмерим с размером области подвижности фиксированного знака, т.е., грубо говоря, за один акт носитель уйдет из области, например, положительной $K(\varepsilon)$ в область отрицательной $K(\varepsilon)$. Заметим, что чисто формально, при достаточно больших $\varepsilon_{0}$, перескок за один акт $\Delta(1)=4\left(\sqrt{\varepsilon_{0}}-1\right) \ll \varepsilon_{0}$, т. е. носители относительно слабо меняют энергию за один акт, и, значит, столкновения можно считать упругими. Однако такой упругости оказывается недостаточно: за один перескок $K(\varepsilon)$ тоже должна измениться незначительно, что в случае рассеяния носителей на акустических фононах не имеет места. Физическая интерпретация $K(\varepsilon)$, предложенная в [13], становится неприменимой, так как она неявно основывалась на том, что за много столкновений квазиподвижность носителей не должна сильно меняться. В нашем примере за один акт она может не только сильно измениться по величине, но и вообще поменять знак.

Проведем оценку энергии носителя, при которой пренебрежение конечностью $\Theta_{\mathrm{D}}$ в интеграле столкновений на акустических фононах законно. Для этого нужно, чтобы энергия испущенного или поглощенного фонона была значительно меньше энергии Дебая.

Из (29) и рис. 3 видно, что частицы с начальной энергией $\varepsilon_{0}$ за один элементарный акт меняют ее не больше чем на $\max \left(\varepsilon_{0}-\left(\sqrt{\varepsilon_{0}}-2\right)^{2},\left(\sqrt{\varepsilon_{0}}+2\right)^{2}-\varepsilon_{0}\right)=$ $=4\left(\sqrt{\varepsilon_{0}}+1\right)$. Значит, должно выполняться $\Theta_{\mathrm{D}}>4$ $\times\left(\sqrt{\varepsilon_{0}}+1\right) \Leftrightarrow \varepsilon_{0}<\left(\Theta_{\mathrm{D}} / 4-1\right)^{2}$, где $\Theta_{\mathrm{D}}$, также как и $\varepsilon_{0}$, обезразмеренна на $m s^{2} / 2$.

Например, в алмазе $\Theta_{\mathrm{D}} \approx 2000 \mathrm{~K}$, для тяжелых дырок и продольных акустических фононов $m_{p, h}=1.08 m_{c}$, $S_{\mathrm{LA}}=1.833 \cdot 10^{6} \mathrm{~cm} / \mathrm{c}[20]$, откуда легко получаем оценку $\varepsilon_{0}<1600$. В наших расчетах $K(\varepsilon)$ считалась до $\varepsilon \approx 600-700$, значит, в этом случае пренебрежение конечностью $\Theta_{\mathrm{D}}$ в интеграле столкновений законно.

Необходимо также отметить, что при достаточно высоких температурах (в алмазе при $T \approx 300 \mathrm{~K}$ ) на подвижность начинает существенно влиять рассеяние на оптических фононах, которое в нашей модели не учитывалось.

\section{6. Заключение}

Введено понятие квазиподвижности, обобщающее характеристику носителей заряда, предложенную Хаксли и Кромптоном, на случай неупругого рассеяния, и позволяющее в стационарном случае посчитать интегральную подвижность. В приближении времени релаксации получена формула сложения квазиподвижностей, обусловленных разными физическими механизмами.

Рассмотрена функция обезразмеренной квазиподвижности носителей заряда в твердых телах при рассеянии на акустических фононах, показана универсальность этой функции, рассмотрены ее ключевые особенности, в том числе чередование зон положительной и отрицательной квазиподвижности при достаточно низких температурах. Уточнен критерий возможности понимания квазиподвижности как подвижности ансамбля носителей определенной энергии. 
Полученные результаты важны для численных расчетов и обработки экспериментальных данных по кинетике носителей заряда в твердых телах.

Работа поддержана грантом Министерства образования и науки РФ (проектная часть государственного задания, проект № 3.679.2014/K). Работа выполнена при частичной поддержке Правительства РФ (соглашение № 05.Y09.21.0018).

\section{Список литературы}

[1] M.A. Prelas, G. Popovici, L.K. Bigelow (eds). Handbook of Industrial Diamonds and Diamond Films (N.Y., Marcel Deccer, 1998).

[2] S. Koizumi, C.E. Nebel, M. Nesladek (eds). Physics and Applications of CVD Diamond. (Weinheim WILEY-VCH Verlag GmbH \& Co.KGaA, 2008).

[3] R.S. Balmer, I. Friel, S.M. Woollard, C.J.H. Wort, G.A. Scarsbrook, S.E. Coe, H. El-Hajj, A. Kaiser, A. Denisenko, E. Kohn, J. Isberg. Phil. Trans. R. Soc. A, 366, 251 (2008).

[4] R.S. Sussmann. CVD Diamond for Electronic Devices and Sensors. (Wiley, Wiley Series in Materials for Electronic \& Optoelectronic Applications, 2009).

[5] V.D. Blank, V.S. Bormashov, S.A. Tarelkin, S.G. Buga, M.S. Kuznetsov, D.V. Teteruk, N.V. Kornilov, S.A. Terentiev, A.P. Volkov. Diamond Relat. Mater., 57, 32 (2015).

[6] Т.Н. Мамедов, А.Г. Дутов, Д. Герлах, В.Н. Горелкин, К.И. Грицай, В.А. Жуков, А.В. Стойков, В.Б. Шипило, У. Циммерманн. Препринт ОИЯИ (Дубна, 2004) Р142004-104.

[7] T.N. Mamedov, D. Andreika, A.S. Baturin, D. Herlach, V.N. Gorelkin, K.I. Gritsaj, V.G. Ralchenko, A.V. Stoykov, V.A. Zhukov, U. Zimmermann. Physica B, 374-375, 390 (2006).

[8] В.Ф. Елесин. УФН, 175, 197 (2005).

[9] А.С. Александров, Ю.А. Быковский, В.Ф. Елесин, Е.А. Протасов, А.Г. Родионов. Письма ЖЭТФ, 12, 57 (1970).

[10] А.С. Александров, Ю.А. Быковский, В.Ф. Елесин, Е.А. Протасов, А.Г. Родионов. Письма ЖЭТФ, 64, 231 (1973).

[11] А.С. Александров, А.Н. Кулямзин, А.П. Менушенков, Е.А. Протасов, П.А. Черемных. ФТТ, 19 (5), 1518 (1977).

[12] A.S. Baturin, V.N. Gorelkin, V.S. Rastunkov, V.R. Soloviev. Physica B, 374-375, 340 (2006).

[13] Л. Хаксли, Р. Кромптон. Диффузия и дрейф электронов в газах (М., Мир, 1977) с. 86, 198.

[14] А.С. Батурин, В.Н. Горелкин, В.Р. Соловьев, И.В. Черноусов. Физика плазмы, 34, 431 (2008).

[15] А.С. Батурин, В.Н. Горелкин, В.Р. Соловьев, И.В. Черноусов. ФТП, 44 (7), 897 (2010).

[16] Ю.М. Белоусов, В.Р. Соловьев, И.В. Черноусов. ФТП, 47 (12), 1630 (2013).

[17] И.А. Варфоломеев, В.Н. Горелкин, В.Р. Соловьев. Тр. МФТИ, 5 (3), 139 (2013).

[18] Yu.M. Belousov, I.V. Chernousov, V.R. Soloviev, I.A. Varfolomeev. Proc. 2nd Int. Conf. Photonics, Optics and Laser Technology (7-9 January, Lisbon, Portugal, 2014) p. 122.

[19] R.D. White, R.E. Robson, B. Schmidt, M.A. Morrison. J. Phys. D: Appl. Phys., 36, 3125 (2003).
[20] O. Madelung. Semiconductors: Data Handbook. (Berlin, Heidelberg, Springer-Verlag, 2004).

Редактор Г.А. Оганесян

\section{On mobility of definite energy charge carriers}

\author{
Yu.M. Belousov, V.N. Gorelkin, I.V. Chernousov \\ Moscow Institute of Physics and Technology \\ (State University), \\ 141701 Dolgoprudniy, Russia
}

Abstract We consider a function of quasi-mobility of charge carriers with given energy for their dynamics description with the help of kinetic equation in an important case of isotropic two-term approximation. In stationary case this function is independent of charge carriers' source function, and allows one calculating integral mobility. The influence of system parameters on quasi-mobility function is analyzed. It is shown that generally this function does not describe a contribution of carriers with given energy to total mobility. It is known that in case of almost elastic scattering quasimobility function can have a clear physical meaning, but in case of carriers scattering on acoustic phonons in solids this interpretation proves to be wrong due to peculiarities of collision integral and quasi-mobility function. 